\title{
The GAD-7 and the PHQ-8 exhibit the same mathematical pattern of item responses in the general population: analysis of data from the National Health Interview Survey
}

Shinichiro Tomitaka ${ }^{1,2^{*}}$ and Toshiaki A. Furukawa ${ }^{1}$

\begin{abstract}
Background: Recent studies have shown that, among the general population, responses to depression-rating scales follow a common mathematical pattern. However, the mathematical pattern among responses to the items of the Generalized Anxiety Disorder-7 (GAD-7) is currently unknown. The present study investigated whether item responses to the GAD-7, when administered to the general population, follow the same mathematical distribution as those of depression-rating scales.

Methods: We used data from the 2019 National Health Interview Survey (31,997 individuals), which is a nationwide survey of adults conducted annually in the United States. The patterns of item responses to the GAD-7 and the Patient Health Questionnaire-8 (PHQ-8), respectively, were analyzed inductively.

Results: For all GAD-7 items, the frequency distribution for each response option ("not at all,"'several days,"'more than half the days," and "nearly every day," respectively) was positively skewed. Line charts representing the responses to each GAD-7 item all crossed at a single point between "not at all" and "several days" and, on a logarithmic scale, showed a parallel pattern from "several days" to "nearly every day."This mathematical pattern among the item responses was identical to that of the PHQ-8. This characteristic pattern of the item responses developed because the values for the "more than half the days" to "several days" ratio were similar across all items, as were the values for the "nearly every day" to "more than half the days" ratio.
\end{abstract}

Conclusions: Our results suggest that the symptom criteria of generalized anxiety disorder and major depression have a common distribution pattern in the general population.

Keywords: Generalized anxiety disorder, GAD-7, Depressive symptoms, PHQ-8, Item response, Mathematical model

\section{Background}

Generalized anxiety disorder (GAD) is one of the most common mental disorders [1]. Among the general population, its 12-month prevalence and lifetime morbid risk

*Correspondence: tomitaka.shinichiro@jp.panasonic.com

2 Department of Mental Health, Panasonic Health Center, Landic Building

3F, Nishishinbashi 3-8-3, Minato-ku, Tokyo 105-0003, Japan

Full list of author information is available at the end of the article are estimated to be $2.0 \%$ and $9.0 \%$, respectively [2, 3]. Several screening instruments have been developed for effectively identifying probable cases of $\operatorname{GAD}[4,5]$. In particular, the Generalized Anxiety Disorder-7 (GAD-7) is one of the most commonly used tools for GAD screening worldwide $[6,7]$. The GAD-7 measures GAD based on the associated symptom criteria listed in the Diagnostic and Statistical Manual of Mental Disorders, Fourth Edition [4]. It is valid and reliable, with a sensitivity of 
$89 \%$, a specificity of $82 \%$, and a high Cronbach Alpha value $(0.9)[8,9]$. The distribution pattern, among the general population, of responses to the GAD-7 symptom criteria, is of interest because, although the diagnosis of GAD is based on responses to these symptom criteria, the mathematical pattern of responses to the GAD-7 items in the context of the general population is currently unknown.

Major depression is another common mental disorder; among the general population, its 12-month prevalence and lifetime morbid risk are estimated to be $8.6 \%$ and $29.9 \%$, respectively [2]. Recent analyses of large-scale national survey data have shown that responses to scale items concerning depressive symptoms exhibit a common mathematical pattern among the general population. In an analysis of data from a Japanese national survey in which the Center for Epidemiologic Studies Depression Scale (CES-D) was administered to members of the general population, the present authors found that responses to 16 depressive-symptom items exhibit a common mathematical pattern among the general population $[10,11]$. Figure 1A shows the relative frequency of each response for each of the 16 items. In this figure, it can be seen that the lines cross between "rarely" and "a little of the time," with most of them meeting at a single point on the graph; then, they begin to converge between "a little of the time" and "all of the time." Meanwhile, on a logarithmic scale, the converging lines show a parallel pattern from "a little of the time" to "all of the time" (Fig. 1B) [11]. The existence of a common mathematical pattern in responses to depression-rating scale items has been confirmed for a considerable number of nationally representative survey datasets worldwide; for example, CES-D data from the Irish Longitudinal Study on Ageing [12], 12-item General Health Questionnaire data from the Eurobarometer surveys [13], nine-item Patient Health Questionnaire (PHQ-9) data from the National Health and Nutrition Examination Survey in the United States [14], and six-item Kessler Psychological Distress Scale data from the national survey of Midlife Development [15] and the National Health Interview Survey in the United States [16]. This strongly suggests that the reproducibility of the item-response pattern is high. Moreover, it is noteworthy that rating scales other than depression-rating scales do not show such a mathematical pattern among the general population [10].

There are two primary reasons why identifying the mathematical pattern of responses to the GAD-7 is important. First, if the item responses to the GAD-7 and depression-rating scales show the same mathematical pattern, this will contribute to improving understanding of the relationship between GAD-7 and depressionrating scales. In general, the mathematical pattern of a sampling distribution reflects the mechanism by which the variables are generated [17]. Thus, if the GAD-7 and depression-rating scales share a common mathematical
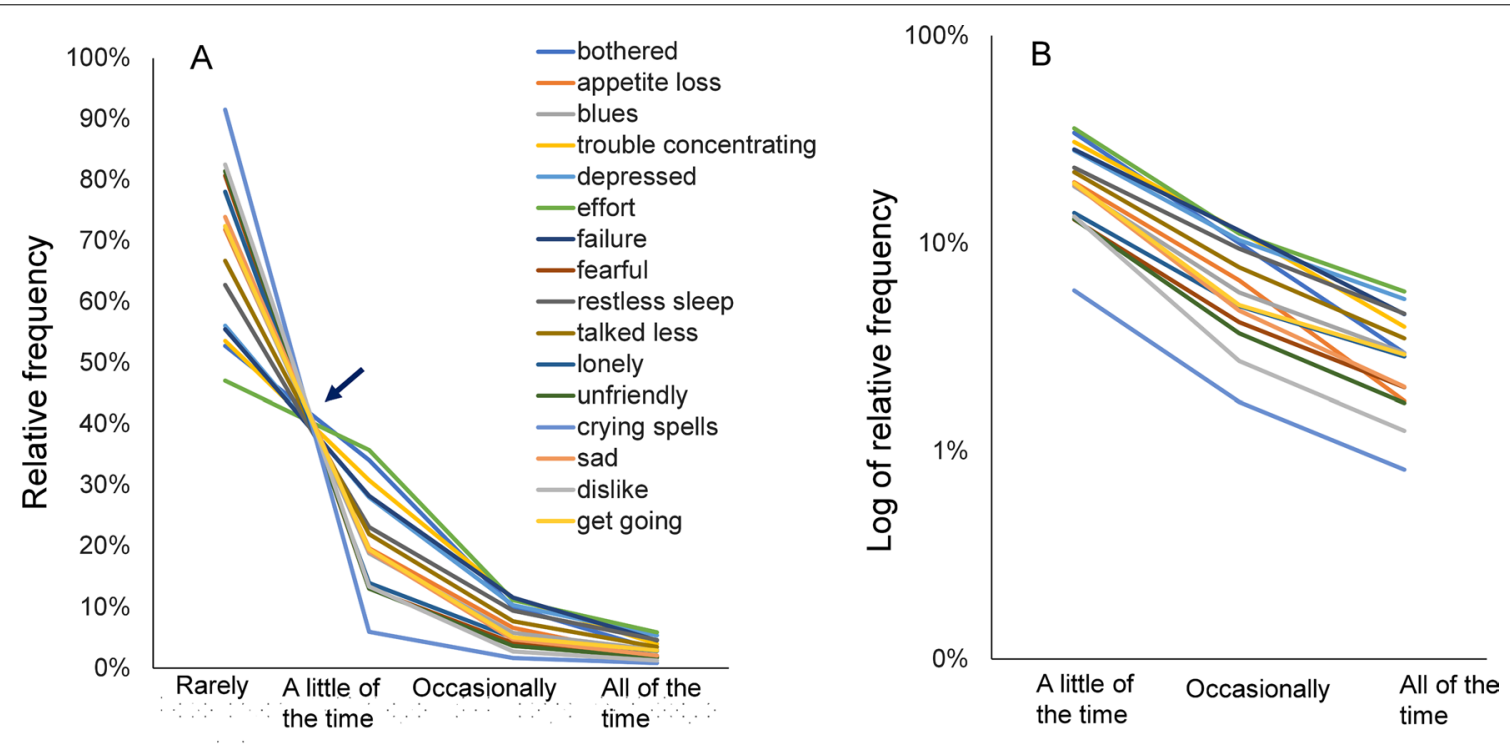

Fig. 1 Responses to the 16 depressive-symptom items of the Center for Epidemiologic Studies Depression Scale. Responses to the 16 items are presented using a normal scale (A), and a logarithmic scale (B). A The lines for the item responses appear to cross at a single point between "rarely" and "a little of the time," whereas they display a converging pattern between "a little of the time" and "all of the time." B On a logarithmic scale, the lines for the 16 items exhibit a parallel linear pattern from "a little of the time" to "all of the time." Image used under CC-BY license: PLoS ONE, https:// doi.org/10.1371/journal.pone.0165928.g001 
pattern in terms of item responses, this will suggest that the symptom criteria of GAD and major depression have a common distribution mechanism. Such a relationship is somewhat likely, as GAD-7 and depression-rating scales are known to be strongly linked; studies have reported moderate to strong correlations between the GAD-7 and depression-rating scales [4, 18, 19]. Moreover, numerous studies have reported high comorbidity of GAD and depression [1, 20-22]. Second, if a mathematical pattern is identified among the item responses to the GAD-7, this would help conduct statistical procedures. Parametric statistics that assume normality are widely used to analyze rating-scale data [23]; however, there is currently no evidence that the item responses to the GAD-7 follow a normally distributed latent variable. Thus, to conduct statistical procedures regarding the GAD-7, it is essential to determine whether the variables feature a mathematical pattern [24].

Generally, larger datasets enable researchers to better analyze sampling distribution patterns. The National Health Interview Survey (NHIS) is conducted annually in the United States and is designed to provide nationally representative estimates for a range of health status variables [25]. In 2019, the NHIS employed the GAD-7 and the eight-item Patient Health Questionnaire (PHQ-8) for the first time in its long history [26]. The PHQ-8 has good validity and reliability for detecting major depression, with a sensitivity of $88 \%$, a specificity of $88 \%$, and a high Cronbach's Alpha value (0.89) [27]. As a result of the large sample size and limited selection bias involved, the GAD-7 and PHQ-8 data collected through the NHIS are suitable for clarifying the aforementioned issue regarding determining the mathematical pattern of the GAD-7.

Using data from the 2019 edition of the NHIS, the present study investigated the respective mathematical patterns of the item responses to the GAD-7 and PHQ-8 when administered to members of the general population. In particular, we sought to determine whether responses to anxiety-rating scales exhibit the same mathematical pattern as those to depression-rating scales. Furthermore, we built a distribution model for the item responses for the GAD-7.

\section{Methods}

\section{Dataset}

We used data from the 2019 edition of the NHIS. The NHIS, which is conducted annually by the National Center for Health Statistics, is designed to survey the health of the general population of the United States and obtain nationally representative estimates of certain health variables [28]. The NHIS targets the civilian noninstitutionalized population of the United States. A sample of households is created, and from each participating family, one "sample adult" aged 18 years or older is randomly selected and invited to participate in the survey. In 2019, the final response rate was 59.1\% [28]. The datasets analyzed during the present study are available from the NHIS repository [25]; NHIS data are available to researchers worldwide.

The sample comprised 31,997 respondents (ages $18-19$ years: $\mathrm{n}=530$ [male: $\mathrm{n}=263$ ]; ages $20-29$ years: $\mathrm{n}=3944$ [male: $\mathrm{n}=1,881$ ]; ages $30-39$ years: $\mathrm{n}=5178$ [male: $\mathrm{n}=2393$ ]; ages $40-49$ years: $\mathrm{n}=4656$ [male: $\mathrm{n}=2238$ ]; ages $50-59$ years: $\mathrm{n}=5,282$ [male: $\mathrm{n}=2573$ ]; ages $60-69$ years: $\mathrm{n}=5921$ [male: $\mathrm{n}=2707$ ]; ages $70-79$ years: $n=4072$ [male: $n=1766$ ]; ages 80 years and older: $\mathrm{n}=2414$ [male: $\mathrm{n}=912$ ]). The sociodemographic characteristics of the 2019 NHIS sample are reported in detail elsewhere [26].

\section{Ethics statement}

The present study used de-identified data that are available to the public. The ethics committees of Kyoto University and Panasonic Health Center do not consider the analysis of de-identified public data to represent research of human subjects. These committees ruled that institutional review board approval was unnecessary for the present research.

\section{Measures}

The 2019 NHIS questionnaires included the GAD-7 and the PHQ-8. The GAD-7 comprises seven items that are based on the symptom criteria of GAD listed in the DSM-5 [4]. In the 2019 NHIS, respondents were asked how often they had experienced each symptom during the past two weeks. Each item was self-rated using four-point response options: "not at all," "several days," "more than half the days," and "nearly every day," which were scored as $0,1,2$, and 3, respectively. Meanwhile, the PHQ-8 comprises eight items and is designed to assess major depressive disorder, also based on the DSM-5's associated symptom criteria [29]. The PHQ-8 is very similar to the PHQ-9 but omits item 9 of the PHQ-9 ("How often have you been bothered by thoughts that you would be better off dead or hurting yourself in some way?"). PHQ-8 items are answered using the same response scale as that used for the GAD-7.

\section{Analysis}

First, we analyzed the pattern of item responses to the PHQ-8 to identify whether any common characteristics were present. As the 2019 NHIS was a survey with a complex design, analyses of item response frequencies were weighted by survey-specific weights which made the sample representative of the target population [8]. Previous studies have reported that, when a self-report scale 
for measuring depression is administered among the general population, the ratios between consecutive response options remain similar across all items in the scale, except for the option at the lower end of the score range [30]. Thus, the respective ratios of "more than half the days" to "several days" and "nearly every day" to "more than half the days" were calculated for all eight items of the PHQ8. Thereafter, we graphically analyzed the mathematical pattern of item responses to the PHQ-8. Similarly, we analyzed the respective ratios of "more than half the days" to "several days," and "nearly every day" to "more than half the days" for the GAD-7. Thereafter, we graphically investigated whether the item responses to the GAD-7 followed the same pattern as those of the PHQ-8. These analyses were performed by sex. Correlations were examined between the GAD-7 and the PHQ-8.

Based on the consequent finding that the values for the "more than half the days" to "several days" ratio and the values for the "nearly every day" to "more than half the days" ratio were similar across all items of the GAD7, we built an inductive model of the item responses to the GAD-7. Analyses were conducted using IBM SPSS complex samples for Windows, version 27 (IBM Corp., Armonk, N.Y., USA).

\section{Results}

\section{Demographic characteristics of the participants}

Of the 31,997 respondents, those who did not respond to all items of the PHQ- 8 and the GAD-7 $(3.3 \%, n=1043)$ were excluded from this analysis. The final sample consequently comprised 30,954 respondents (14,262 males; ages 18-19: $\mathrm{n}=515$ [male, $\mathrm{n}=254$ ]; ages $20-29: \mathrm{n}=3840$ [male, $\mathrm{n}=1841$ ]; ages 30-39: $\mathrm{n}=5036$ [male, $\mathrm{n}=2327$ ]; ages 40-49: $\mathrm{n}=4512$ [male, $\mathrm{n}=2175$ ]; ages 50-59: $\mathrm{n}=5107$ [male; $\mathrm{n}=2479$ ]; ages $60-69: \mathrm{n}=5741$ [male, $\mathrm{n}=2628$ ]; ages 70-79: $\mathrm{n}=3933$ [male, $\mathrm{n}=1693$ ]; and age 80 or older: $\mathrm{n}=2270$ [male, $\mathrm{n}=865$ ]).

\section{Correlation among the 15-item scores of the GAD-7 and the PHQ-8}

Although to varying degrees, all items of the PHQ-8 and the GAD-7 had moderate-to-strong positive Spearman's correlations $(\rho=0.30-0.71)$ (Additional file 1: Table S1). The Pearson's correlation between the total GAD-7 scores and the PHQ-8 scores was 0.79 .

\section{PHQ-8 item responses}

Table 1 displays the response rates for the PHQ- 8 items. A common tendency was observed for all seven items, with the frequency being highest for "not at all," decreasing from "not at all" to "more than half the days," and increasing from "more than half the days" to "nearly every day." There were no exceptions to this tendency. The rates of "more than half the days" to "several days" and "nearly every day" to "more than half the days" were $0.23 \pm 0.04$, and $1.52 \pm 0.17$, respectively. The standard deviations of the two rates were rather small when compared with the averages, which suggests that the two rates were similar, to some extent, across the eight items.

Each of the eight items was scored using a four-point scale: 0 (indicating "not at all"), 1 ("several days"), 2 ("more than half the days"), and 3 ("nearly every day"). Average rate data are presented as mean \pm standard deviation.

To assess the pattern among the item responses for the PHQ-8, line graphs representing the response frequencies for each item were plotted onto a single graph (Fig. 2). As indicated by the arrow shown in Fig. 2A, the lines for all eight items appeared to cross at a single point between "not at all" and "several days." Conversely, from "several days" to "more than half the days" the lines

Table 1 Item responses to the PHQ-8

\begin{tabular}{|c|c|c|c|c|c|c|}
\hline \multirow[t]{2}{*}{ Item } & \multicolumn{4}{|l|}{ Item responses } & \multirow{2}{*}{$\begin{array}{l}\text { Rate of more than half } \\
\text { the days to several days }\end{array}$} & \multirow{2}{*}{$\begin{array}{l}\text { Rate of nearly every day to } \\
\text { more than half the days }\end{array}$} \\
\hline & Not at all (\%) & $\begin{array}{l}\text { Several } \\
\text { days (\%) }\end{array}$ & $\begin{array}{l}\text { More than half } \\
\text { the days (\%) }\end{array}$ & $\begin{array}{l}\text { Nearly } \\
\text { every day } \\
(\%)\end{array}$ & & \\
\hline 1. Loss of interest or pleasure & 83.2 & 11.1 & 2.4 & 3.3 & 0.21 & 1.38 \\
\hline 2. Depressed mood & 83.4 & 11.9 & 2.1 & 2.6 & 0.18 & 1.27 \\
\hline 3. Sleep disturbances & 68.5 & 18.4 & 4.7 & 8.4 & 0.26 & 1.79 \\
\hline 4. Anergia or fatigability & 60.0 & 25.7 & 5.4 & 8.8 & 0.21 & 1.61 \\
\hline 5. Appetite disturbances & 82.7 & 10.3 & 2.8 & 4.2 & 0.27 & 1.52 \\
\hline 6. Low self-esteem & 86.8 & 8.9 & 1.8 & 2.5 & 0.20 & 1.42 \\
\hline 7. Concentration difficulties & 88.1 & 7.4 & 1.7 & 2.8 & 0.23 & 1.69 \\
\hline 8. Psychomotor symptoms & 93.7 & 3.7 & 1.0 & 1.5 & 0.28 & 1.47 \\
\hline Average & 80.8 & 12.2 & 2.7 & 4.3 & $0.23 \pm 0.04$ & $1.52 \pm 0.17$ \\
\hline
\end{tabular}


A

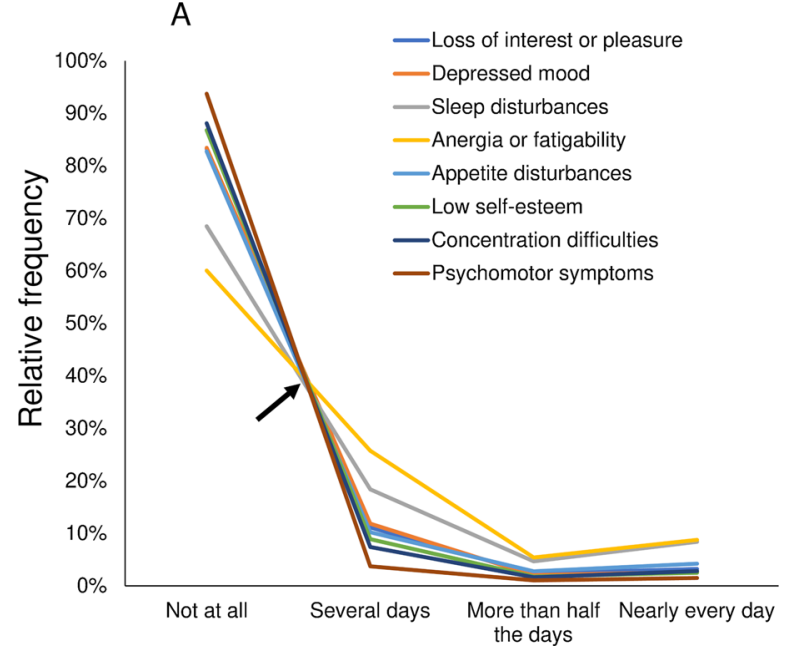

B

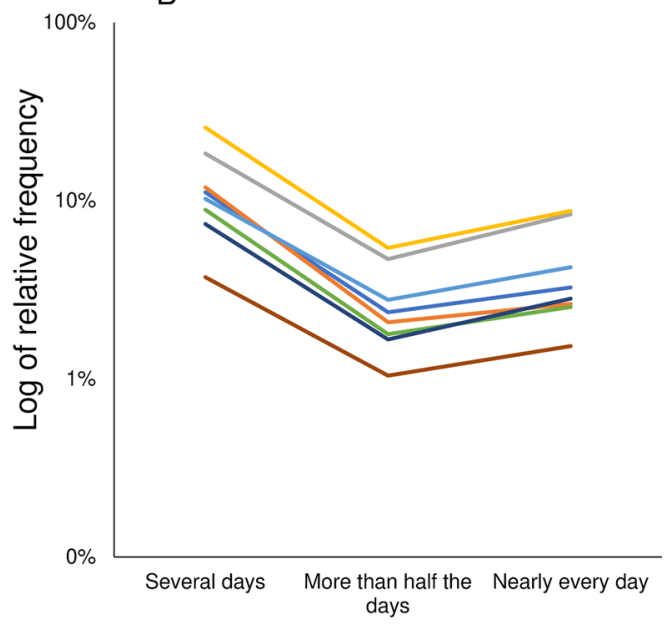

Fig. 2 Responses to the eight depressive-symptom items of the Patient Health Questionnaire-8. The responses to the eight items are presented using a normal scale (A), and a logarithmic scale (B). A As indicated by the arrow, the line graphs of the eight items appear to cross at a single point between "not at all" and "several days." The lines decrease in synchrony from "several days" to "more than half the days," before increasing in synchrony from "more than half the days" to "nearly every day." B The lines show parallel fluctuation from "several days" to "nearly every day."

decreased in synchrony, before increasing in synchrony from "more than half the days" to "nearly every day."

When presented on a logarithmic scale (Fig. 2B), the lines showed a generally parallel pattern from "several days" to "nearly every day." Mathematically, this parallelism of the eight lines on the logarithmic scale reflects the similarity of the values for the "more than half the days" to "several days" ratio across the eight items, and of the values for the "nearly every day" to "more than half the days" ratio across the items, respectively [30].
This supports the abovementioned observation that these ratios were similar, to some extent, among all items (Table 1). Taken together, the graphical analysis confirmed that the item responses exhibited a common pattern across the eight items.

\section{Item responses to the GAD-7}

Table 2 shows the item response rates for the GAD-7. The responses for all seven items showed a similar pattern, with the frequency being highest for "not at all,"

Table 2 Item responses to the GAD-7

\begin{tabular}{|c|c|c|c|c|c|c|}
\hline \multirow[t]{2}{*}{ Item } & \multicolumn{4}{|c|}{ Item responses (\%) } & \multirow{2}{*}{$\begin{array}{l}\text { Rate of more than half } \\
\text { the days to several } \\
\text { days }\end{array}$} & \multirow{2}{*}{$\begin{array}{l}\text { Rate of nearly every day to } \\
\text { more than half the days }\end{array}$} \\
\hline & Not at all (\%) & $\begin{array}{l}\text { Several } \\
\text { days } \\
(\%)\end{array}$ & $\begin{array}{l}\text { More than } \\
\text { half the days } \\
(\%)\end{array}$ & $\begin{array}{l}\text { Nearly } \\
\text { every day } \\
(\%)\end{array}$ & & \\
\hline $\begin{array}{l}\text { 1. Feeling nervous, anxious, or on } \\
\text { edge }\end{array}$ & 76.5 & 16.8 & 2.9 & 3.8 & 0.17 & 1.30 \\
\hline $\begin{array}{l}\text { 2. Not being able to stop or control } \\
\text { worrying }\end{array}$ & 83.3 & 10.5 & 2.3 & 3.9 & 0.21 & 1.74 \\
\hline $\begin{array}{l}\text { 3. Worrying too much about differ- } \\
\text { ent things }\end{array}$ & 76.3 & 16.3 & 2.8 & 4.6 & 0.17 & 1.64 \\
\hline 4. Trouble relaxing & 80.7 & 12.6 & 2.5 & 4.2 & 0.20 & 1.64 \\
\hline $\begin{array}{l}\text { 5. Being so restless that it is hard to } \\
\text { sit still }\end{array}$ & 88.6 & 7.2 & 1.5 & 2.7 & 0.20 & 1.87 \\
\hline $\begin{array}{l}\text { 6. Becoming easily annoyed or } \\
\text { irritable }\end{array}$ & 74.9 & 18.1 & 3.3 & 3.8 & 0.18 & 1.15 \\
\hline $\begin{array}{l}\text { 7. Feeling afraid as if something } \\
\text { awful might happen }\end{array}$ & 87.8 & 8.2 & 1.6 & 2.4 & 0.20 & 1.49 \\
\hline Average & 81.1 & 12.8 & 2.4 & 3.6 & $0.19 \pm 0.02$ & $1.55 \pm 0.25$ \\
\hline
\end{tabular}


decreasing from "not at all" to "more than half the days," and increasing from "more than half the days" to "nearly every day"; this was consistent with the rate distribution among the PHQ- 8 items. The respective rates of "more than half the days" to "several days" and "nearly every day" to "more than half the days" were $0.19 \pm 0.02$, and $1.55 \pm 0.25$, respectively, also similar to those of the PHQ-8 (0.23 \pm 0.04 , and $1.52 \pm 0.17$, respectively). Moreover, the standard deviations of the two rates were rather small in comparison to the averages, again consistent with the results of the PHQ-8.

Each of the seven items is scored on a four-point scale: 0 (indicating "not at all"), 1 ("several days"), 2 ("more than half the days"), and 3 ("nearly every day"). Average rate data are presented as mean \pm standard deviation.

To identify the patterns of the item responses, line graphs representing the response frequencies for each item were plotted on the same scale (Fig. 3). Consistent with the results of the PHQ-8, the responses exhibited a common pattern across the seven items of the GAD-7. As indicated by the arrow shown in Fig. 3A, the lines for the eight items appeared to cross at a single point between "not at all" and "several days." The lines for the seven items then decreased in synchrony from "several days" to "more than half the days," before increasing in synchrony from "more than half the days" to "nearly every day." When compared to the line graphs for the responses to the PHQ-8 (Fig. 2A), the seven lines representing the responses to the GAD-7 seemed to overlap to a greater extent (Fig. 3A).

When presented on a logarithmic scale, the lines for each item response showed a generally parallel pattern from "several days" to "nearly every day" (Fig. 3B). The gradient of the linear patterns of item responses decreases from "several days" to "more than half the days," and then increases from "more than half the days" to "nearly every day." While, between "several days" and "more than half the days," the lines appear to follow an almost perfect parallel pattern, between "more than half the days" and "nearly every day" the pattern is less parallel. These observations accord with the finding that the standard deviation of the ratio of "more than half the days" to "several days" (0.02) was smaller when compared to that of "nearly every day" to "more than half the days" $(0.25)$.

\section{Common pattern in all responses to the PHQ-8 and GAD-7}

To confirm whether all item responses to the PHQ-8 and GAD-7 show the same mathematical pattern, all of these item responses were plotted on the same scale (Fig. 4). As indicated by the arrow shown in Fig. 4A, the lines for all 15 items appeared to cross at a single point between "not at all" and "several days." Conversely, the lines decreased in synchrony from "several days" to "more than half the days," before increasing in synchrony from "more than half the days" to "nearly every day."

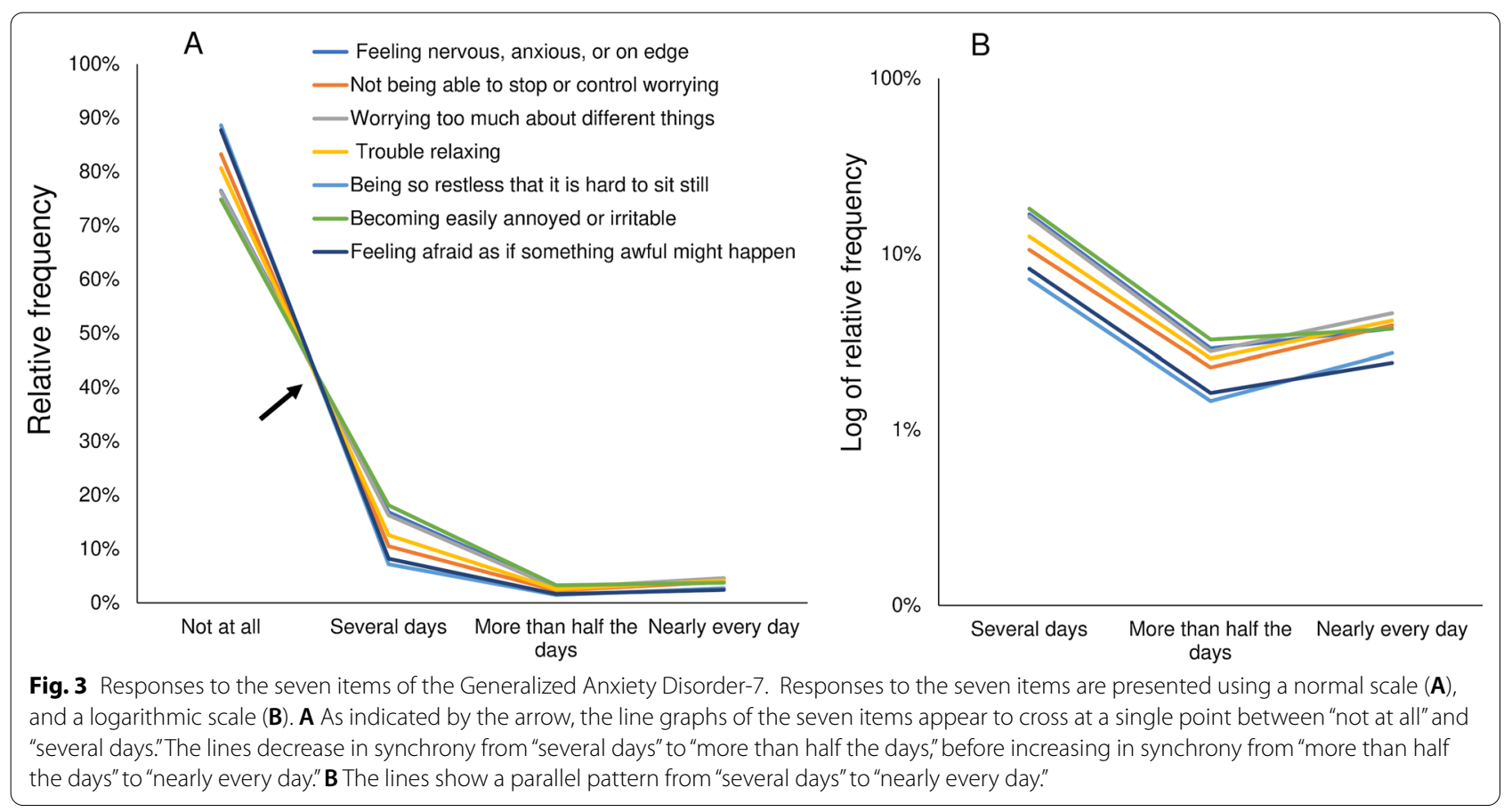



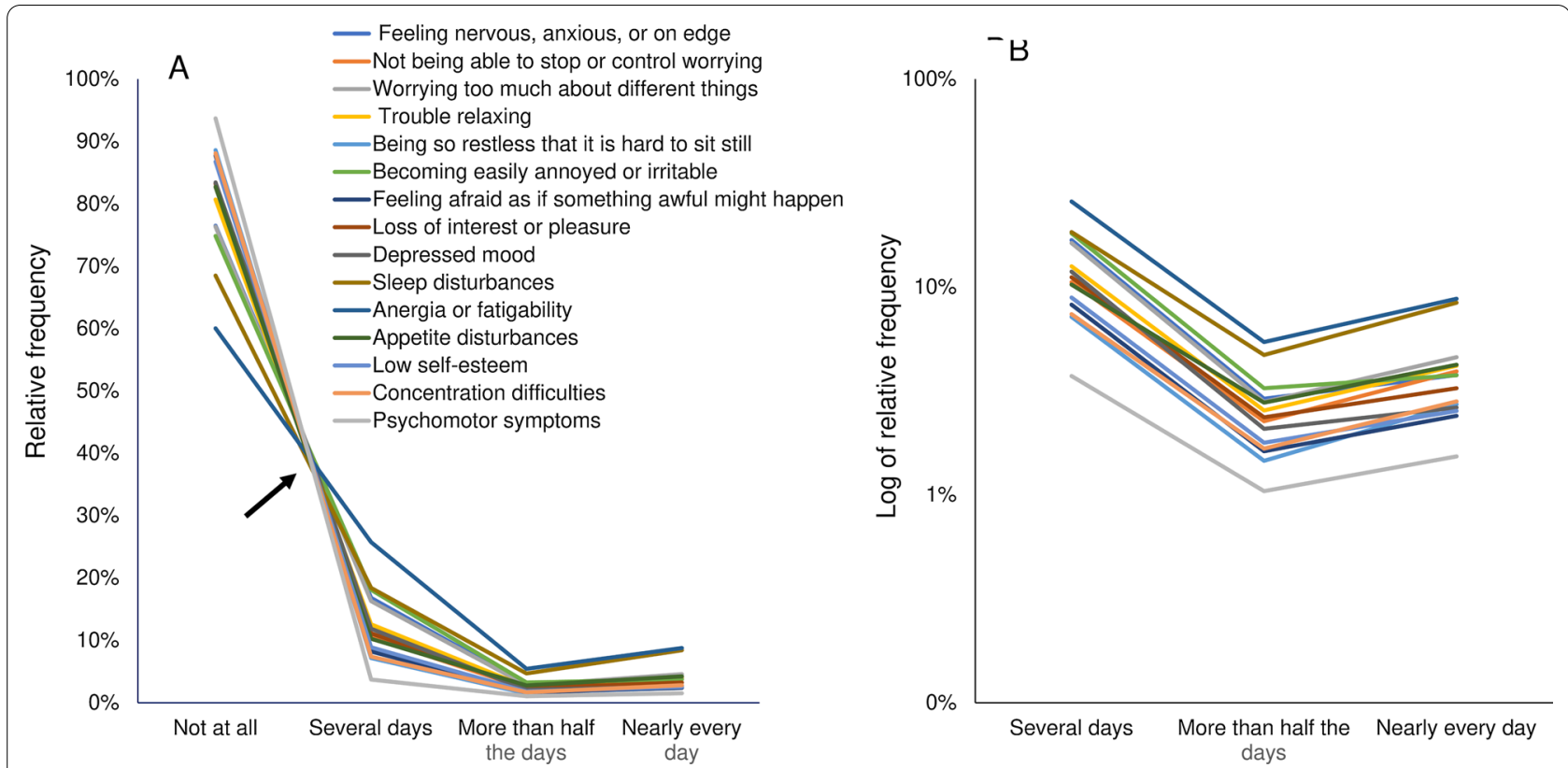

Fig. 4 Responses to the 15 items of the Patient Health Questionnaire-8 and the Generalized Anxiety Disorder-7. Responses to the 15 items are presented using a normal scale (A), and a logarithmic scale (B). A As indicated by the arrow, the line graphs of the seven items appear to cross at a single point between "not at all" and "several days."The lines decrease in synchrony from "several days" to "more than half the days," before increasing in synchrony from "more than half the days" to "nearly every day." B The lines show parallel fluctuation from "several days" to "nearly every day."

When presented on a logarithmic scale, the lines for the 15 item responses showed a generally parallel pattern from "several days" to "nearly every day" (Fig. 4B). Taken together, these graphs indicated that all responses to the PHQ-8 and GAD-7 show a common mathematical pattern.

To confirm whether all item responses to the PHQ-8 and GAD-7 showed the same mathematical pattern regardless of sex, we performed graphical analyses. For both males (Additional file 2: Fig. S1) and females (Additional file 3: Fig. S2), the item responses of the GAD-7 and the PHQ-8 followed the same mathematical distribution.

\section{Mathematical model of the item responses for the GAD-7 and PHQ-8}

Based on the finding that the values for the "more than half the days" to "several days" ratio were similar across all items of the PHQ- 8 and GAD-7, and that the values for the "nearly every day" to "more than half the days" ratio were also similar across these items, we built a mathematical model for the item responses for these scales.

The blue line shown in Fig. 5A illustrates the pattern of the model of the item responses for such scales. For a four-point scale such as the GAD-7, when the relative frequency of "several days," the ratio of "more than half the days" to "several days," and the ratio of "nearly every day" to "more than half the days" are presented as $\mathrm{P}_{1}, \mathrm{r}_{1}$, and $r_{2}$, respectively, the relative frequencies of "not at all," "several days," "more than half the days," and "nearly every day" are expressed as $1-\mathrm{P}_{1} \times\left(1+\mathrm{r}_{1}+\mathrm{r}_{1} \mathrm{r}_{2}\right), \mathrm{P}_{1}$, $\mathrm{P}_{1} \mathrm{r}_{1}$, and $\mathrm{P}_{1} \mathrm{r}_{1} \mathrm{r}_{2}$, respectively (Fig. 5A).

As shown in Fig. 5B, the relative frequencies of "not at all," "several days," "more than half the days," and "nearly every day" for another item (the red line) are expressed as $1-\mathrm{P}_{2} \times\left(1+\mathrm{r}_{1}+\mathrm{r}_{1} \mathrm{r}_{2}\right), \mathrm{P}_{2}, \mathrm{P}_{2} \mathrm{r}_{1}$, and $\mathrm{P}_{2} \mathrm{r}_{1} \mathrm{r}_{2}$, respectively. According to the calculation, the intersection between "not at all" and "several days", is expressed as follows: $(\mathrm{x}, \mathrm{y})=\left(\frac{\mathrm{r} 1 \mathrm{r} 2+\mathrm{r} 1+1}{\mathrm{r} 1 \mathrm{r} 2+\mathrm{r} 1+2}, \frac{1}{\mathrm{r} 1 \mathrm{r} 2+\mathrm{r} 1+2}\right)$. The intersection point is expressed by $r_{1}$ and $r_{2}$ only. Consequently, regardless of the value of $\mathrm{P}_{1}$ or $\mathrm{P}_{2}$, all of the lines cross at a single point between "not at all" and "several days." According to the model of the item responses, it is natural that the lines of all 15 items appear to cross at a single point between "not at all" and "several days" (Fig. 5A), because the respective rates of "more than half the days" to "several days" $\left(\mathrm{r}_{1}\right)$ and "nearly every day" to "more than half the days" $\left(\mathrm{r}_{2}\right)$ were similar across the PHQ-8 and the GAD-7 (Tables 1, 2).

Figure 5C shows a line chart model of the item responses between "several days" and "nearly every day" on a logarithmic scale. On the logarithmic scale, the relative frequencies of "several days," "more than half 


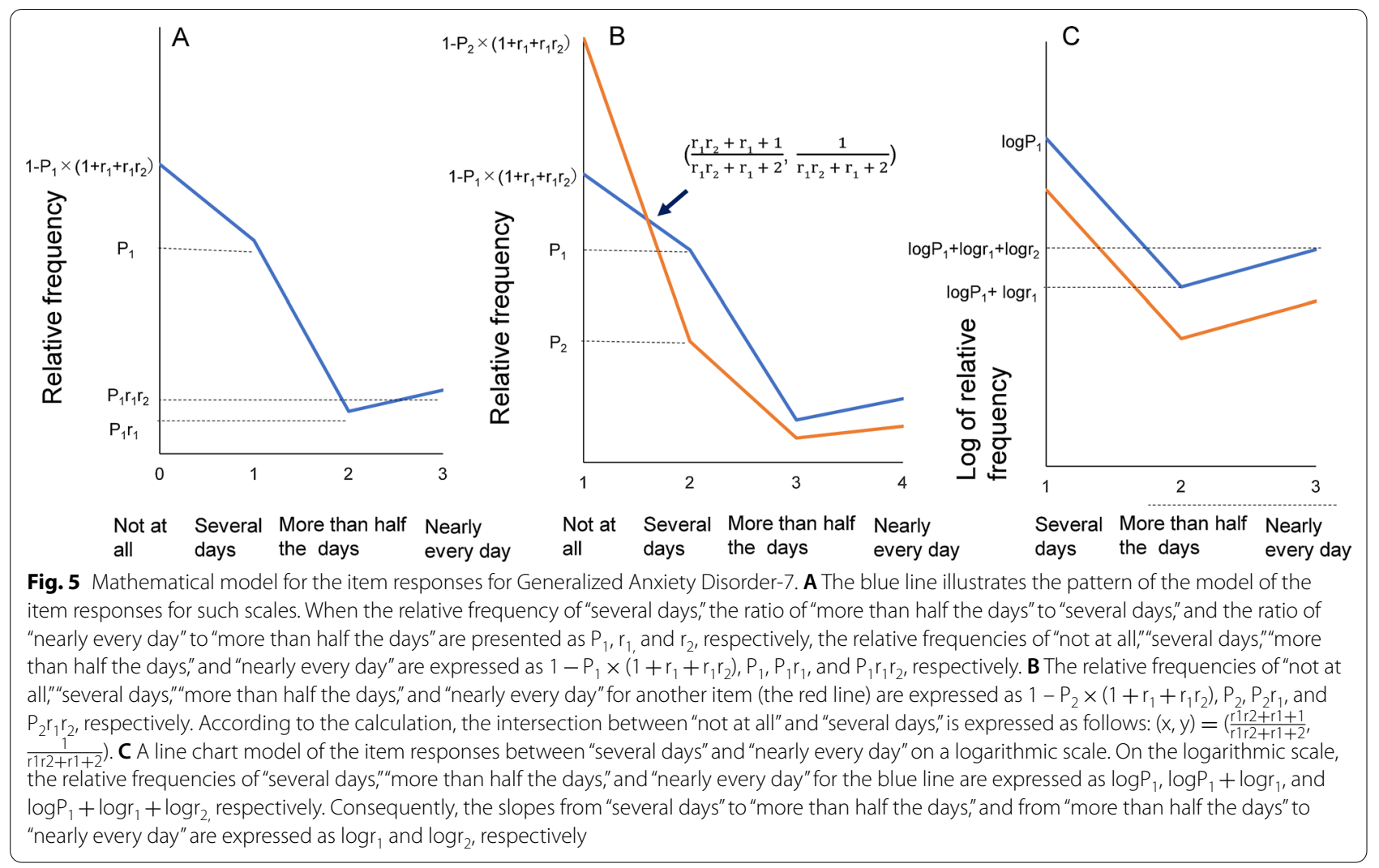

the days," and "nearly every day" for the blue line are expressed as $\log \mathrm{P}_{1}, \log \mathrm{P}_{1}+\log \mathrm{r}_{1}$, and $\log \mathrm{P}_{1}+\log \mathrm{r}_{1}+\log \mathrm{r}_{2}$, respectively. Consequently, the slopes from "several days" to "more than half the days," and from "more than half the days" to "nearly every day" are expressed as $\log r_{1}$ and $\operatorname{logr}_{2}$, respectively. It is noteworthy that the slopes on the logarithmic scale are expressed by $r_{1}$ and $r_{2}$ only. Therefore, regardless of the value of $\mathrm{P}_{1}$ or $\mathrm{P}_{2}$, all the lines show a parallel pattern between "several days" and "nearly every day" on a logarithmic scale.

\section{Discussion}

In this study, we found a common mathematical pattern among responses to the seven GAD-7 items when this scale is administered to the general population. The pattern was characterized by the lines crossing at a single point between the responses "not at all" and "several days," and, on a logarithmic scale, showing a parallel pattern from "several days" to "nearly every day." This pattern was consistent with the corresponding trend for the PHQ-8. A mathematical model of the item responses showed that the characteristic pattern of the item responses developed because the values for the "more than half the days" to "several days" ratio were similar across all items, as were the values for the "nearly every day" to "more than half the days" ratio.
For both the GAD-7 and PHQ-8, differing patterns were observed between the trends for the lower end of the response options and those for the remaining options; this is consistent with findings for the CES-D (Figs. 1, 2, 3). A possible reason for this finding is the psychological process underlying how each symptom item is rated [10]. In general, assessment of each symptom criterion is conducted in two stages. In the first stage, each respondent assesses whether the given symptom criterion is present. If the degree of each symptom criterion does not reach the threshold at which the respondent notices the symptom, it is categorized as "not at all." However, if the degree of the symptom criterion reaches or exceeds this threshold, it is categorized using the remaining response options, such as "several days," "more than half the days," and "nearly every day." This two-stage process implies that "not at all" corresponds to the under-threshold range, while the remaining degreeadverb options correspond to the over-threshold range. If each of the remaining response options corresponds to a specific proportion of the over-threshold range, the item responses should show different patterns between the response option at the lower end and the remaining options [30]. Further research should focus on how each of the remaining response options can correspond to a specific proportion of the over-threshold range. 
The item responses for the GAD-7 and PHQ-8 exhibit the same mathematical pattern. This suggests that symptoms of GAD and major depression share a common distribution mechanism. Supporting this hypothesis, the 15 items of the PHQ-9 and the GAD-7 had moderateto-strong positive correlations in this study. Moreover, numerous studies have reported that GAD and depression have high comorbidity [1, 20-22]. The present authors have previously shown that a characteristic pattern of item responses occurs only when a general trait of the variables in question follows an exponential distribution [30].

When compared to the graph for the PHQ-8 (Fig. 2A), the seven lines in the graph representing the responses to the GAD-7 overlapped to a greater extent (Fig. 3A). In fact, for the GAD-7 the frequency of "not at all" ranged from 74.9 to $88.6 \%$ (Table 2), whereas for the PHQ-8 the frequency of "not at all" ranged from 60.0 to $93.7 \%$ (Table 1). This difference between the GAD-7 and the PHQ-8 regarding the range of "not at all" may reflect a difference in how the items of each scale were selected by the scale developers. The symptom criteria measured in the GAD-7 were selected by rank ordering items based on their respective correlations with the total score for a larger scale that reflected all of the symptom criteria for GAD contained in the Diagnostic and Statistical Manual of Mental Disorders, Fourth Edition [4]. In contrast, the PHQ-8 includes a wide range of symptoms, such as psychological, somatic, and social symptoms. The criterion symptoms of the PHQ-8 derive from the Feighner criteria for depression, which emphasize consideration of the multifaceted nature of symptoms rather than rank ordering of the correlations of each symptom [31].

On a logarithmic scale, the lines for the PHQ-8 and the GAD-7 items showed a stronger parallel pattern between "several days" and "more than half the days" when compared to the pattern between "more than half the days" and "nearly every day" (Figs. 2, 3). This finding accords with those of previous investigations involving the PHQ-9 and the CES-D [10, 14]. A possible explanation for this difference is the sample sizes in question; supporting this possible explanation is the fact that the relative frequencies of "more than half the days" and "nearly every day" were much smaller than that of "several days."

For both sexes, the item responses for the GAD-7 and PHQ-8 exhibited the same mathematical pattern. Previous analyses have reported that the item responses for depression rating scales show the same mathematical pattern regardless of age and nationality [13, 32]. Taken together, item responses on the GAD-7 and depression rating scales may follow the same characteristic pattern regardless of demographic factors, such as sex, age, and nationality. However, it is unknown whether the GAD-7 and depressive symptom scales follow the same mathematical distribution in a clinical population. A future study with more focus on a clinical population is therefore suggested.

This study has several limitations. First, we did not investigate whether the findings were generalizable to symptom criteria associated with other anxiety disorders. Extensive additional research is necessary to generalize the findings to such symptoms. Second, this study lacks quantification of the goodness of fit of the model presented. When determining the fit of established unitary models (i.e., normal, linear, and quadratic models), established methods can be used. However, the present model is unique and complicated. Moreover, a unified descriptor for interpreting the goodness of fit does not exist yet. Therefore, we were unable to describe the degree of the present model's fit using unified descriptors, such as "slightly," "moderately," and "strongly." Further research is necessary to quantify the fit of the present model. Third, because of the cross-sectional nature of the data, we could not examine the temporal sequencing of the distribution pattern of depression and anxiety symptoms. Several studies suggest that anxiety disorders tend to temporally precede depression [33-35]. Further longitudinal studies are necessary to clarify the temporal sequencing of the distribution pattern of depression and anxiety symptoms. Finally, an important limitation of this research is the representativeness of the study sample; we performed a complete-case analysis which could have induced response bias. Another strategy for handling missing data is multiple imputation, which simulates the missing data based on theory. However, most multiple imputation softwares assume that data are normally distributed. Since the variables of our data are not normally distributed, there is the statistical concern that multiple imputation can induce bias [36]. In addition, the final response rate of the NHIS survey was $59.1 \%$. This could also have induced bias because the characteristics of nonresponders may differ from responders [37].

Despite the above limitations, this study also has several strengths. First, the use of data from the NHIS meant that a large sample size with limited selection bias was analyzed. Second, although the present study employed a simple analysis approach (visualization using line charts), it enabled us to identify a complex pattern of item responses; graphical analysis is useful for exploratory data analysis of complex models [38]. Third, our observation of a mathematical pattern of item responses in data representing a large sample size is noteworthy because distributional models are needed to conduct statistical procedures. Finally, this is the first report on the mathematical pattern among item responses to the GAD-7 when this scale is administered to the general population. 
The fact that the item responses to the GAD-7 and the PHQ-8 exhibit the same mathematical distribution provides additional insight into the mechanism of these scales. From the viewpoint of public health, the distribution pattern of item responses on the GAD-7 is necessary to analyze the distribution of anxiety conditions in defined populations. The mathematical model for item responses on the GAD-7 enables us to easily describe the distribution of anxiety symptoms with parameters. Moreover, as noted previously, the observed distribution patterns provide evidence that item responses on the GAD-7 follow a non-normal distribution in the general population, suggesting that statistical methods assuming normality require careful consideration when analyzing such data. More research should be undertaken to further explore how item responses to such scales show a common mathematical pattern among the general population.

\section{Conclusions}

The findings of this study provide evidence that there is a common mathematical pattern among the item responses to the GAD-7. Given that the item responses to the GAD-7 exhibit the same mathematical distribution as those of the PHQ-8, we conjecture that symptoms of GAD and major depression share a common distribution mechanism. This finding that the item responses to the GAD-7 and the PHQ-8 exhibit the same mathematical distribution provides further insight into the relationship between anxiety and depression.

\section{Abbreviations}

CES-D: Center for Epidemiologic Studies Depression Scale; DSM-5: Diagnostic and Statistical Manual of Mental Disorders, Fifth Edition; GAD: generalized anxiety disorder; GAD-7: Generalized Anxiety Disorder Scale; NHIS: National Health Interview Survey; PHQ: patient health questionnaire.

\section{Supplementary Information}

The online version contains supplementary material available at https://doi. org/10.1186/s40359-021-00657-9.

Additional file 1. Correlation among the 15-item scores of the GAD-7 and the $\mathrm{PHQ}-8$.

Additional file 2: Fig. S1. Responses to the 15 items of the Patient Health Questionnaire-8 and the Generalized Anxiety Disorder-7 in males. Responses by males to the 15 items are presented using a normal scale (A) and a logarithmic scale (B). A As indicated by the arrow, the line graphs of the 15 items appear to cross at a single point between "not at all" and "several days."The lines decrease in synchrony from "several days" to "more than half the days," before increasing in synchrony from "more than half the days" to "nearly every day."

Additional file 3: Fig. S2. Responses to the 15 items of the Patient Health Questionnaire-8 and the Generalized Anxiety Disorder-7 in females. Responses by females to the 15 items are presented using a normal scale $(\mathbf{A})$ and a logarithmic scale (B). A As indicated by the arrow, the line graphs of the 15 items appear to cross at a single point between "not at all" and "several days."The lines decrease in synchrony from "several days" to "more than half the days," before increasing in synchrony from "more than half the days" to "nearly every day."

\section{Acknowledgements}

We would like to thank the National Health Interview Survey for providing the data for this study.

\section{Authors' contributions}

ST contributed to the conception and design of the study, the analysis and interpretation of data, and wrote the manuscript. TAF contributed to the design of the study and revision of the manuscript. Both authors read and approved the final manuscript.

\section{Funding}

This work was supported by JSPS KAKENHI, Japan [Grant Number: 21K03105].

\section{Availability of data and materials}

The datasets analyzed in the present study are available from the National Health Interview Survey's official repository: https://www.cdc.gov/nchs/nhis/ nhis_2019_data_release.htm.

\section{Declarations}

Ethics and consent to participate

The present study analyzed de-identified data that are available to the public. The ethics committees of Kyoto University Graduate School of Medicine and Panasonic Health Center do not consider secondary analyses of publicly available data to represent research on human subjects.

\section{Consent for publication}

Not applicable.

\section{Conflict of interest}

The authors declare that they have no competing interests.

\section{Author details}

${ }^{1}$ Department of Health Promotion and Human Behavior, Department of Clinical Epidemiology, School of Medicine/School of Public Health, Kyoto University Graduate, Yoshida Konoe-cho, Sakyo-ku, Kyoto 606-8501, Japan. ${ }^{2}$ Department of Mental Health, Panasonic Health Center, Landic Building 3F, Nishishinbashi 3-8-3, Minato-ku, Tokyo 105-0003, Japan.

Received: 17 April 2021 Accepted: 17 September 2021

Published online: 23 September 2021

\section{References}

1. Kessler RC, Gruber M, Hettema JM, Hwang I, Sampson N, Yonkers KA. Co-morbid major depression and generalized anxiety disorders in the National Comorbidity Survey follow-up. Psychol Med. 2008;38:365-74. https://doi.org/10.1017/S0033291707002012.

2. Kessler RC, Petukhova M, Sampson NA, Zaslavsky AM, Wittchen $\mathrm{H}-\mathrm{U}$. Twelve-month and lifetime prevalence and lifetime morbid risk of anxiety and mood disorders in the United States. Int J Methods Psychiatr Res. 2012;21:169-84. https://doi.org/10.1002/mpr.1359.

3. Andrews G, Henderson S, Hall W. Prevalence, comorbidity, disability and service utilisation. Br J Psychiatry. 2001;178:145-53. https://doi.org/10. 1192/bjp.178.2.145

4. Spitzer RL, Kroenke K, Williams JBW, Löwe B. A brief measure for assessing generalized anxiety disorder. Arch Intern Med. 2006;166:1092. https://doi. org/10.1001/archinte.166.10.1092.

5. Newman MG, Zuellig AR, Kachin KE, Constantino MJ, Przeworski A, Erickson $T$, et al. Preliminary reliability and validity of the generalized anxiety disorder questionnaire-IV: a revised self-report diagnostic measure of 
generalized anxiety disorder. Behav Ther. 2002;33:215-33. https://doi.org/ 10.1016/S0005-7894(02)80026-0.

6. Hinz A, Klein AM, Brähler E, Glaesmer H, Luck T, Riedel-Heller SG, et al. Psychometric evaluation of the generalized anxiety disorder screener GAD-7, based on a large German general population sample. J Affect Disord. 2017;210:338-44. https://doi.org/10.1016/j.jad.2016.12.012.

7. Doi S, Ito M, Takebayashi Y, Muramatsu K, Horikoshi M. Factorial validity and invariance of the 7-item Generalized Anxiety Disorder Scale (GAD-7) among populations with and without self-reported psychiatric diagnostic status. Front Psychol. 2018;9:1741. https://doi.org/10.3389/fpsyg.2018. 01741.

8. NHIS - 2019 NHIS. https://www.cdc.gov/nchs/nhis/2019nhis.htm. Accessed 5 Jun 2021.

9. Tiirikainen K, Haravuori H, Ranta K, Kaltiala-Heino R, Marttunen M. Psychometric properties of the 7-item Generalized Anxiety Disorder Scale (GAD7) in a large representative sample of Finnish adolescents. Psychiatry Res. 2019;272:30-5. https://doi.org/10.1016/j.psychres.2018.12.004.

10. Tomitaka S, Kawasaki Y, Furukawa T. A distribution model of the responses to each depressive symptom item in a general population: a cross-sectional study. BMJ Open. 2015:5:e008599. https://doi.org/10.1136/bmjop en-2015-008599.

11. Tomitaka S, Kawasaki Y, Ide K, Akutagawa M, Yamada H, Furukawa TA, et al: Relationship between item responses of negative affect items and the distribution of the sum of the item scores in the general population. PLoS ONE. 2016;11:e0165928. https://doi.org/10.1371/journal.pone.0165928.

12. Tomitaka S, Kawasaki Y, Ide K, Akutagawa M, Ono Y, Furukawa TA. Distribution of item responses and total item scores for the Center for Epidemiologic Studies Depression Scale (CES-D): data from the Irish Longitudinal Study on Ageing (TILDA). PLoS ONE. 2018;13:e0202607. https://doi.org/ 10.1371/journal.pone.0202607.

13. Tomitaka S, Kawasaki Y, Ide K, Akutagawa M, Ono Y, Furukawa TA. Responses to depressive symptom items exhibit a common mathematical pattern across the European populations. Sci Rep. 2019;9:14923. https://doi.org/10.1038/s41598-019-51499-w.

14. Tomitaka S, Kawasaki Y, Ide K, Akutagawa M, Yamada H, Ono Y, et al. Distributional patterns of item responses and total scores on the PHQ-9 in the general population: data from the National Health and Nutrition Examination Survey. BMC Psychiatry. 2018;18:108. https://doi.org/10. 1186/s12888-018-1696-9.

15. Tomitaka S, Kawasaki Y, Ide K, Akutagawa M, Yamada H, Yutaka O, et al. Pattern analysis of total item score and item response of the Kessler Screening Scale for Psychological Distress (K6) in a nationally representative sample of US adults. PeerJ. 2017;5:e2987. https://doi.org/10.7717/ peerj.2987.

16. Tomitaka S, Kawasaki Y, Ide K, Akutagawa M, Ono Y, Furukawa TA. Further evidence that item responses on the Kessler Psychological Distress Scale exhibit the characteristic pattern in the general population. Heliyon. 2019;5:e01387. https://doi.org/10.1016/j.heliyon.2019.e01387.

17. Hand DJ. Principles of data mining. Drug Saf. 2007;30:621-2. https://doi. org/10.2165/00002018-200730070-00010.

18. Kroenke K, Wu J, Yu Z, Bair MJ, Kean J, Stump T, et al. Patient Health questionnaire anxiety and depression scale. Psychosom Med. 2016;78:716-27. https://doi.org/10.1097/PSY.0000000000000322.

19. Teymoori A, Gorbunova A, Haghish FE, Real R, Zeldovich M, Wu Y-J, et al. Factorial structure and validity of depression (PHQ-9) and anxiety (GAD-7) scales after traumatic brain injury. J Clin Med. 2020;9:873. https://doi.org/ $10.3390 / \mathrm{jcm} 9030873$.

20. Schoevers RA, Beekman ATF, Deeg DJH, Jonker C, Tilburg W van. Comorbidity and risk-patterns of depression, generalised anxiety disorder and mixed anxiety-depression in later life: results from the AMSTEL study. Int J Geriatr Psychiatry. 2003;18:994-1001. https://doi.org/10.1002/gps.1001.
21. Lamers F, van Oppen P, Comijs HC, Smit JH, Spinhoven P, van Balkom AJLM, et al. Comorbidity patterns of anxiety and depressive disorders in a large cohort study. J Clin Psychiatry. 2011;72:341-8. https://doi.org/10. 4088/JCP.10m06176blu.

22. Johansson R, Carlbring P, Heedman Å, Paxling B, Andersson G. Depression, anxiety and their comorbidity in the Swedish general population: point prevalence and the effect on health-related quality of life. Peer. 2013;1:e98. https://doi.org/10.7717/peerj.98.

23. Jamieson S. Likert scales: how to (ab)use them. Med Educ. 2004:38:12178. https://doi.org/10.1111/j.1365-2929.2004.02012.x.

24. Micceri T. The unicorn, the normal curve, and other improbable creatures. Psychol Bull. 1989;105:156-66. https://doi.org/10.1037/0033-2909.105.1. 156.

25. Centers for Disease Control and Prevention. NHIS—National Health Interview Survey Homepage. 2017. https://www.cdc.gov/nchs/nhis/index. htm. Accessed 4 Sep 2017.

26. NHIS - 2019 Questionnaire Redesign. https://www.cdc.gov/nchs/nhis/ 2019_quest_redesign.htm. Accessed 21 Feb 2021.

27. Kroenke K, Spitzer RL, Williams JBW, Löwe B. The patient health questionnaire somatic, anxiety, and depressive symptom scales: a systematic review. Gen Hosp Psychiatry. 2010;32:345-59.

28. CDC - 2015 BRFSS Survey Data and Documentation. https://www.cdc. gov/brfss/annual_data/annual_2015.html. Accessed 11 Aug 2017.

29. Kroenke K, Strine TW, Spitzer RL, Williams JBW, Berry JT, Mokdad AH, et al. The PHQ-8 as a measure of current depression in the general population. J Affect Disord. 2009;114:163-73. https://doi.org/10.1016/j.jad.2008.06. 026.

30. Tomitaka S. Patterns of item score and total score distributions on depression rating scales in the general population: evidence and mechanisms. Heliyon. 2020;6:e05862. https://doi.org/10.1016/j.heliyon.2020.e05862.

31. Kendler KS, Muñoz RA, Murphy G. The development of the feighner criteria: a historical perspective. Am J Psychiatry. 2010;167:134-42. https://doi. org/10.1176/appi.ajp.2009.09081155.

32. Tomitaka S, Kawasaki Y, Ide K, Akutagawa M, Ono Y, Furukawa TA. Agerelated changes in item responses to the patient health questionnaire-9: evidence from the national health and nutrition examination survey. Front Psychiatry. 2020;11:1. https://doi.org/10.3389/fpsyt.2020.00723.

33. Starr LR, Davila J. Temporal patterns of anxious and depressed mood in generalized anxiety disorder: a daily diary study. Behav Res Ther. 2012;50:131-41. https://doi.org/10.1016/j.brat.2011.11.005.

34. Moffitt TE, Harrington H, Caspi A, Kim-Cohen J, Goldberg D, Gregory AM, et al. Depression and generalized anxiety disorder. Arch Gen Psychiatry. 2007;64:651. https://doi.org/10.1001/archpsyc.64.6.651.

35. de Graaf R, Bijl R V., Spijker J, Beekman ATF, Vollebergh WAM. Temporal sequencing of lifetime mood disorders in relation to comorbid anxiety and substance use disorders. Soc Psychiatry Psychiatr Epidemiol. 2003;38:1-11. https://doi.org/10.1007/s00127-003-0597-4.

36. Sterne JAC, White IR, Carlin JB, Spratt M, Royston P, Kenward MG, et al. Multiple imputation for missing data in epidemiological and clinical research: potential and pitfalls. BMJ (Online). 2009;339:157-60. https://doi. org/10.1136/bmj.b2393.

37. Kessler RC, Little RJA, Groves RM. Advances in strategies for minimizing and adjusting for survey nonresponse. Epidemiol Rev. 1995;17:192-204. https://doi.org/10.1093/oxfordjournals.epirev.a036176.

38. Tukey JW. Exploratory data analysis. Boston: Addison-Wesley Pub. Co: 1977.

\section{Publisher's Note}

Springer Nature remains neutral with regard to jurisdictional claims in published maps and institutional affiliations. 